\title{
Channel Capacity and BER Performance Analysis of MIMO System with Linear Receiver in Nakagami Channel
}

\author{
Samarendra Nath Sur ${ }^{\mathrm{a}^{*}}$, Debj yoti Ghosh ${ }^{\mathrm{b}}$ \\ ${ }^{a, b}$ Sikkim Manipal Institute of Technology, Sikkim Manipal University, Sikkim, India.
}

\begin{abstract}
Multiple input multiple output (MIMO) is one of the key technology to accomplish the goal of the future wireless broadband communication. This paper deals with the bit error rate (BER) and capacity performance of the Vertical Bell laboratories layered space-time (VBALST) MIMO systems with a linear receiver such as minimum mean-square error (MMSE) and zero forcing (ZF) over Nakagami-m channel. Both the BER and the capacity of MIMO systems have been analyzed for different modulation techniques and also with various configurations of MIMO systems. We have also analyzed the VBLAST MIMO system performance in a higher SNR region.
\end{abstract}

Index Terms: MMSE, ZF, V-BLAST, MIMO, Nakagami-m, Channel Capacity, BER.

(C) 2013 Published by MECS Publisher. Selection and/or peer review under responsibility of the Research Association of Modern Education and Computer Science

\section{Introduction}

From the perspective of today's broadband communication scenario, the need of high-capacity and highlyreliable wireless communication has been growing noticeably over the last few decades. Most of the wireless communication systems developed before the mid 1990s may be referred to as single input single output (SISO) systems [1]. Later on more researches have been carried on exploring the possibilities of a MIMO system by exploiting the diversity at both the transmitter and receiver. From the perspective of research growth in MIMO technology, channel modeling, capacity analysis, space-time coding (STC), spatial multiplexing (SM) and multi-user detection techniques are the main point of interest for the researchers. At this age of 4th generation broadband communication, the growing demand of higher data rate under the constraint of limited available bandwidth, multi-input and multiple-output (MIMO) systems offer the possibility of spatial multiplexing which enables very high spectral efficiencies $[2,3,4]$ and also this leads to the development of an appropriate signal processing architecture to support the spectral requirement. Vertical Bell laboratories layered space-time (V-BLAST) $[2,5,6]$ is a MIMO architecture known to have spectral efficiencies of 20-40 bps/Hz at 24-34 dB average SNR without coding [7].

* Corresponding author.

E-mail address: samar.sur@gmail.com 
In the wireless environ ment the electro magnetic wave gets polluted by some physical phenomenon such as absorption, reflection, refraction, diffraction, and scattering etc. Because of the existence of physical object in their propagating path, such as trees, buildings, hills etc. [8]. Because of those phenomena the randomly varying phase, amplitude and the angle of arrival of multipath component added up constructively and destructively and give rise to a rapidly fluctuating signal at the receiver front end.

For the practical purposes different fading channel can be modeled, out of which most important fading distributions are Rayleigh and Rician [9]. Recently Nakagami distribution has been of great interest because Nakagami-m fading channel can be considered as a universal one as it represents various fading condition in a wireless channel $[9,10]$. Nakagami-m fading channel can provide severe conditions than the Rayleigh and Rician model and can be considered to fit into the mobile communication channel [9] .W ith the variation in the Nakagami fading parameter-m a wide range of distribution can be realized. It becomes the Rayleigh distribution when $\mathrm{m}=1$ and for $\mathrm{m}>1$ the Rician fading can be closely approximated. Also it becomes the one-sided Gaussian distribution $(\mathrm{m} \rightarrow 0.5)$, and Nakaga mi-m distribution covers no fading channel as $\mathrm{m}$ goes to infinity $[11,12]$. So analysis of Nakaga mi channel is very important.

In this paper we analyze the capacity and BER performance of the V BLAST MIMO system with MMSE and ZF receiver [13] system in Nakagami-m fading channel and also we present the simulation result to analyze the system performance with the variation in the fading parameter $\mathrm{m}$. We also quantify the BER and capacity performance advantage of the MMSE-V-BLAST over the ZF-V-BLAST.

\section{Mathematical Model}

\subsection{Channel Model}

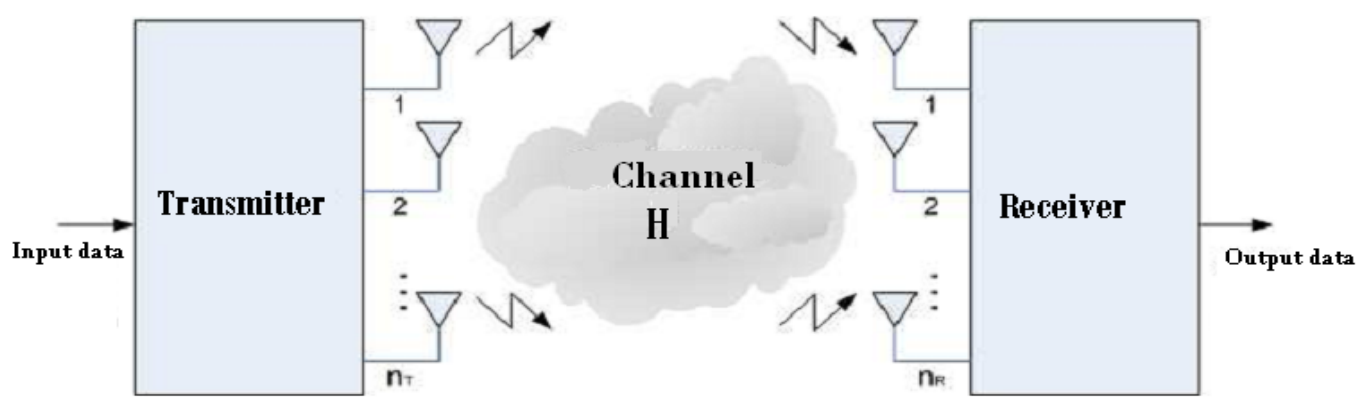

Fig. 1. MIMO system archit ecture.

We consider a MIMO system, as in figure 1, with $N_{t}$ transmitting antenna and $N_{r}$ receiving antennas. The received signal y can be described by

$Y=H x+n$

Where the transmit symbols vector $x$ satisfies $\mathrm{E}\left\{\|\mathrm{x}\|^{2}\right\} \leq \mathrm{P}$ (P is the total power), and $\mathrm{n}$ is the $\mathrm{N}_{\mathrm{r}} \times 1$ additive white Gaussian noise vector. The vector $\mathrm{H}$ represents the slowly vary ing flat fading (Nakagami-m) channels for the wireless transmission. The channel is assumed to be independent and identically distributed (i.i.d) and this follows a Nakagami $-\mathrm{m}$ fading probability distribution function (pdf). Let $\gamma$ represent the instantaneous SNR and it can be defined as 
$\gamma=\beta^{2} \frac{E_{S}}{N_{0}}$

Where $\beta$ is the fading amplitude, $E_{S}$ is the energy per symbol, and $N_{0}$ is the noise spectral density. The probability distribution function of $\beta$ for the Nakagami-m fading channel is given by

$P_{\beta}(\beta)=\frac{2}{\Gamma(m)}\left(\frac{m}{\Omega}\right) \beta^{2 m-1} \exp \left(\frac{-m \beta^{2}}{\Omega}\right), \beta \geq 0, m \geq \frac{1}{2}$

Where $\Gamma($.$) represents the gamma function, \Omega=E\left[\beta^{2}\right]$ and $\mathrm{m}$ is the parameter of fading depth that ranges from 0.5 to infinity and this parameter is responsible for the variation in fading condition.

The fading parameter $\mathrm{m}$ is defined by the equation as given below

$m=\frac{E^{2}\left[\beta^{2}\right]}{\operatorname{var}\left[\beta^{2}\right]}$

Then the pdf of the instantaneous SNR $\gamma$ is given by [14]

$P_{\gamma}(\gamma)=\frac{1}{\Gamma(m)}\left(\frac{m}{\bar{\gamma}}\right)^{m} \gamma^{m-1} \exp \left(\frac{-m \gamma}{\bar{\gamma}}\right), \gamma \geq 0$
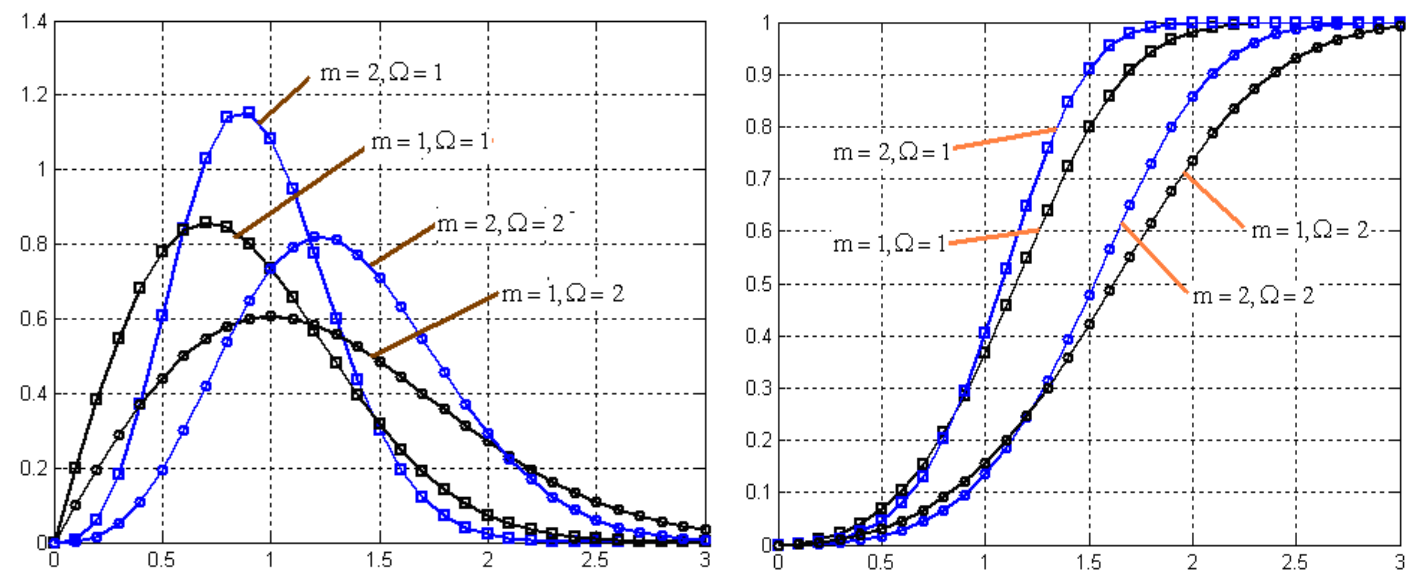

Fig 2. The PDF and CDF plots of Nakagami-m distribution.

\subsection{BER Calculation}

The mo ment generating function (MGF) $[12,15]$ of the SNR in Nakagami-m fading is given by

$\varphi_{\gamma}(s)=\int_{0}^{\infty} \exp (-s \gamma) P_{\gamma}(\gamma) d \gamma=\left(\frac{m}{m+s \gamma}\right)^{m}, m \geq \frac{1}{2}$

The conditional- error probability (CEP) for MSK is given by 
$P_{S}(\gamma)=\frac{1}{\pi} \int_{0}^{\pi-\frac{\pi}{M}} \exp \left(\frac{-\gamma \sin ^{2}\left(\frac{\pi}{M}\right)}{\sin ^{2} \theta}\right) d \theta$

The average symbol error rate in Nakagami channel for positive values of fading depth ' $m$ ' is given by

$$
\begin{aligned}
\bar{P}_{S} & =\int_{0}^{\infty} P_{s}(\gamma) P_{\gamma}(\gamma) d \gamma \\
& =\frac{1}{\pi} I_{S}\left(0, \pi-\frac{\pi}{M},\left(\frac{\bar{y}}{m}\right) \sin ^{2}\left(\frac{\pi}{M}\right), m\right)
\end{aligned}
$$

As in [6], the generalized form of $\mathrm{I}_{\mathrm{S}}$ can be expressed as

$$
\begin{gathered}
I_{S}\left(\theta_{L}, \theta_{U}, c, d\right)=\int_{\theta_{L}}^{\theta_{U}}\left[\frac{\sin ^{2} \theta}{c+\sin ^{2} \theta}\right]^{d} d \theta \\
=\theta_{U}-\theta_{L}-\sqrt{\frac{c}{c+1}}\left[\frac{\pi}{2}+\lambda\left(c, \theta_{U}\right)\right] \sum_{k=0}^{d-1} \frac{1}{[4(c+1)]^{k}}\left\{\left(\begin{array}{c}
2 k \\
k
\end{array}\right)+\frac{1}{\pi} \sum_{h=0}^{k-1}\left(\begin{array}{c}
2 k \\
h
\end{array}\right) \frac{\sin \left[2(k-h) \lambda\left(c, \theta_{U}\right]\right.}{k-h}\right\}+\sqrt{\frac{c}{c+1}}\left[\frac{\pi}{2}+\right. \\
\lambda c \quad, \theta L k=0 d-114 c+1 k 2 k k+1 \pi h=0 k-12 k h \sin 2 k-h \lambda(c, \quad \theta L k-h
\end{gathered}
$$

Where $d$ is a positive integer and

$\lambda(q, \theta)=\tan ^{-1}\left[\sqrt{\left(\frac{q}{1+q}\right)} \cot (\pi-\theta)\right]$

As in [16], from the power series expansion of equation (3) we find that at the higher SNR region the PDF of the fading amplitude $(\beta)$ is inversely vary with the signal SNR level. And the proportionality relation changes with the variation in Nakagami fading shape parameter. For $m=0.5$ (one-sided Gaussian), the PDF changes inversely with SNR levels whereas for $m=1$ (Rayleigh fading) and $m=2$ (Rician fading) it is proportional to the inverse of the square and fifth power of the SNR level respectively. Therefore, the error probability rate in Nakagami fading channel with $m=0.5$ is worse than that of Rayleigh and Rician channel.

\subsection{Linear Receiver:}

\subsubsection{Zero Forcing (ZF):}

The Zero forcing receiver, is a Simple linear receiver, with low computational complexity. Zero forcing implements matrix (pseudo) inverse (+). The ZF estimated received signal is given by:

$\hat{x}=\left(H^{H} H\right)^{-1} H^{H} \cdot x=H^{+} x$

Where superscript $\mathrm{H}$ denotes Hermitian transpose.

And the output SNR for the ZF receiver can be obtained as $\rho_{z f}=\frac{S N R}{\left(H^{H} H\right)^{-1}}$ 
The ZF minimizes interference but suffers from noise enhancement and shapes the received signal, therefore it is free of ISI. The ZF receiver works best with high SNR level.

\subsubsection{Minimum Mean Square Error (MMSE):}

MMSE $[17,18]$ receiver is another type of linear detector which minimizes the mean squared error between the transmitted symbols. MMSE detector helps to jointly minimize both the noise and interference or we can say that the MMSE detector seeks to balance between the cancellation of the interference and reduction of noise enhancement. Therefore MMSE detector outperforms the ZF detector in the presence of noise.

The MMSE receiver gives a solution to:

$\hat{x}=\left(\frac{1}{S N R} I+H^{H} H\right)^{-1} \cdot H^{H} x$

And the output SNR for the ZF receiver can be obtained as

$$
\rho_{\text {mmse }}=\frac{S N R}{\left(H^{H} H+I \frac{1}{S N R}\right)^{-1}}-1
$$

The above two linear equalization algorithm is based on multiplying the received vector by a detection matrix and then decoding the symbols separately. Another approach in VBLAST receiver design is successive interference cancellation to achieve better performance at the cost of much higher complexity.

From the mathematical relation one can estimate that ZF is the limiting form of the MMSE for SNR approaches to infinity. But from the simulation we observed that the bit error rate of MMSE and ZF receiver do not converge even for large SNR. Therefore we find that there is always a SNR gain of MMSE equalizer over the ZF receiver for large SNR values.

\subsection{Capacity Calculation}

The capacity of a MIMO channel can be written as

$C=\log _{2} \operatorname{det}\left(I_{N}+\operatorname{SNR} .\left(H H^{H}\right)\right)$

Where SNR is the average signal to noise ratio at each receiver antenna.

The capacity of a sub-channel in a MIMO system with a linear detector (LD) can be written as

$C_{L D}=\log _{2}\left(I_{N}+S N I R\right)$

For zero forcing receivers, the capacity as sociated with the channel can be written as

$C_{Z F}=\log _{2}\left(1+\frac{S N R}{\left(H^{H} H\right)^{-1}}\right)$

Similarly for MMSE receiver 
$C_{M M S E}=\log _{2}\left(\frac{1}{\left(I_{N}+S N R \cdot H^{H} H\right)^{-\mathrm{T}}}\right)$

As we know that the minimization of the mean square error (MSE) leads to the maximization of the SNR, therefore MMSE based detector achieves higher SNR in comparis on to that of a ZF based detector. And this advantage leads to the gain in channel capacity with MMSE based over that with the ZF based detector.

The capacity gain of MIMO systems with MMSE receiver over the ZF receiver have been analyzed as follows

\section{Then $\mathrm{C}_{\mathrm{MMSE}}-\mathrm{C}_{\mathrm{ZF}}$}

$=\log _{2}\left(\frac{1}{\left(I_{N}+S N R \cdot H^{H} H\right)^{-1}\left(1+\frac{S N R}{\left(H^{H} H\right)^{-1}}\right)}\right)$

For large SNR, $\left(I_{N}+S N R . H^{H} \cdot H\right)^{-1}$ can be expanded as follows

$=\left(S N R \cdot H^{H} H\right)^{-1}\left[1+I_{N}\left(S N R \cdot H^{H} H\right)^{-1}\right]^{-1}$

Using the relation $(1+x)^{-1}=1-x+x^{2}-x^{3}+x^{4}-\ldots \ldots \ldots$ we have

$=\left(S N R \cdot H^{H} H\right)^{-1}-\left[I_{N}\left(S N R \cdot H^{H} H\right)^{-2}\right]+\left[I_{N}{ }^{2}\left(S N R \cdot H^{H} H\right)^{-3}\right]-\ldots \ldots$

$=\left(S N R \cdot H^{H} H\right)^{-1}-\left[I_{N}\left(S N R \cdot H^{H} H\right)^{-2}\right]+O(S N R)^{-3}$

Thus for large SNR level the equation (18) can be written as

$$
\begin{aligned}
& =\log _{2}\left(\frac{1}{\left[\left(S N R \cdot H^{H} H\right)^{-1}-\left[I_{N}\left(S N R \cdot H^{H} H\right)^{-2}\right]+O(S N R)^{-3}\right]\left(1+\frac{S N R}{\left(H^{H} H\right)^{-1}}\right)}\right) \\
& =\log _{2}\left(\frac{S N R}{\left[S N R+\left(H^{H} H\right)^{-1}\right]\left[1-\left\{I_{N}\left(S N R \cdot H^{H} H\right)^{-1}\right\}+O(S N R)^{-2}\right]}\right) \\
& =\log _{2}(S N R)-\log _{2}\left\{1-(S N R)^{-1} I_{N} \cdot\left(H^{H} \cdot H\right)^{-1}+O(S N R)^{-2}\right\}-\log _{2}\left[S N R+\left(H^{H} H\right)^{-1}\right]
\end{aligned}
$$




\section{Result Analysis:}

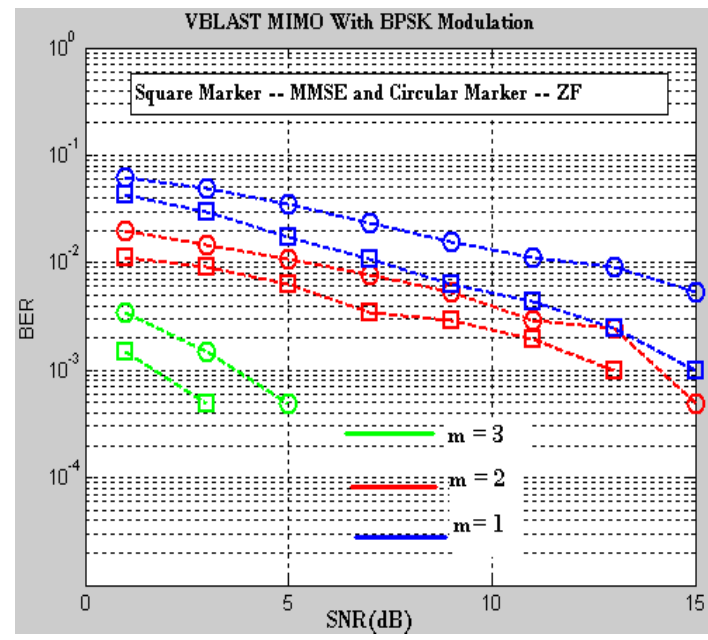

Fig. 3: BERvs. SNR curves for VBLAST MIMO systems in Nakagami channel.

Above figure 3 represents a comparative study between MMSE and ZF receiver in Nakagami channel with variation in fading factor $\mathrm{m}$. With the increase in $\mathrm{m}$, the channel statistic changes and turns AWGN channel for higher values of $\mathrm{m}$. This phenomenon has been established through the simulation as in figure 3 .

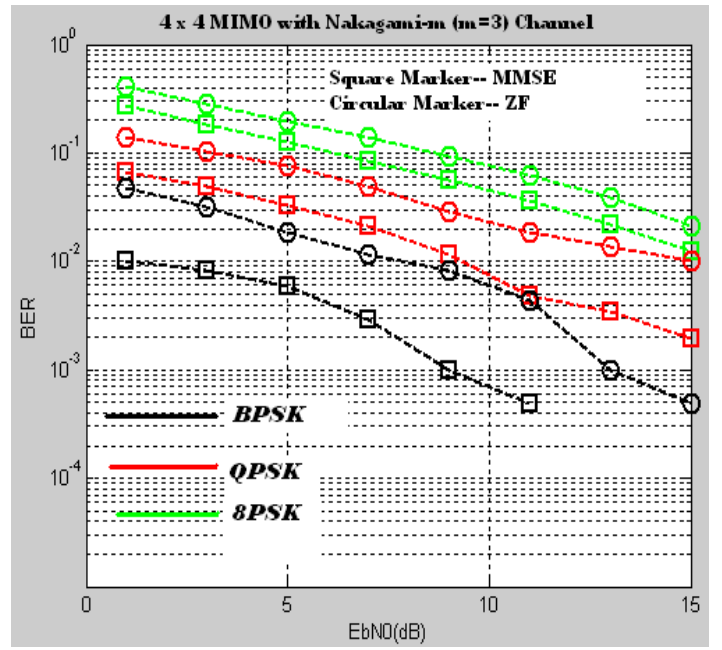

Fig. 4. BER vs. SNR curves for 4 x 4 VBLAST MIMO systems in Nakagami channel with different modulation.

The above figure 4 represents the performance analysis of $4 \times 4$ VBLAST MIMO systems in the Nakagami channel (with fading factor $\mathrm{m}=3$ ) with different digital modulation schemes. 
Table1: BER Comparison Of Different Modulation Techniques.

\begin{tabular}{|c|c|c|c|c|}
\hline \multirow{2}{*}{$\begin{array}{l}\text { Modulation } \\
\text { Techniques }\end{array}$} & \multirow{2}{*}{ Detector } & BPSK & QPSK & 8PSK \\
\cline { 3 - 5 } & & \multicolumn{3}{|c|}{ BER VALUES } \\
\hline \multirow{2}{*}{ SNR=1dB } & MMSE & 0.01025 & 0.06592 & 0.2759 \\
\cline { 2 - 5 } & ZF & 0.04688 & 0.1382 & 0.4106 \\
\hline \multirow{2}{*}{ SNR=11dB } & MMSE & 0.0004883 & 0.004883 & 0.03662 \\
\cline { 2 - 5 } & ZF & 0.004395 & 0.01855 & 0.0625 \\
\hline
\end{tabular}

As in the table it is clear that with the increase in the modulation order the BER performance of the system gets degraded and also the MMSE receiver provide better performance with respect to the ZF receiver. From the theoretical point of view at high SNR level the MSSE receiver tends to behave like a ZF receiver, but from the practical scenario, these two receivers do not fully converge at high SNR level. As in figure 4 with the increase in SNR values the difference in BER level for the above said receivers decrease.

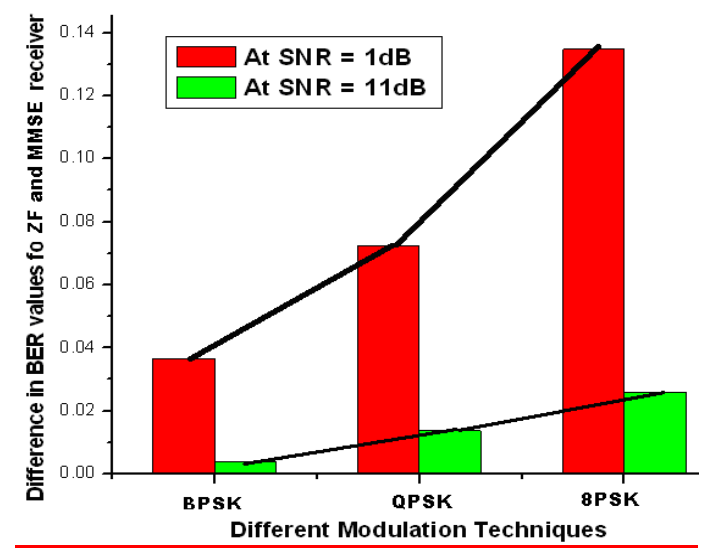

Fig.5: BER Performance comparison of MIMO system in Lower (1 dB) and higher (11dB) SNR region.

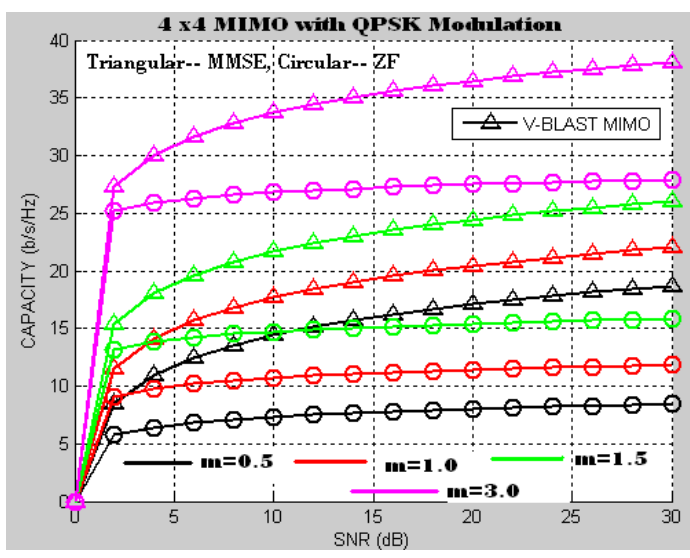

Fig.6. Capacity vs. SNR curves for VBLAST MIMO system in Nakagami channel with QPSK modulation. 
From figure 6 it is clear that with the increase in $\mathrm{m}$ factor value the spectral efficiency of the MIMO systems gets better. And also as MMSE receiver provides better SINR with respect to ZF receiver, it outperforms the ZF receiver in terms of spectral efficiency.

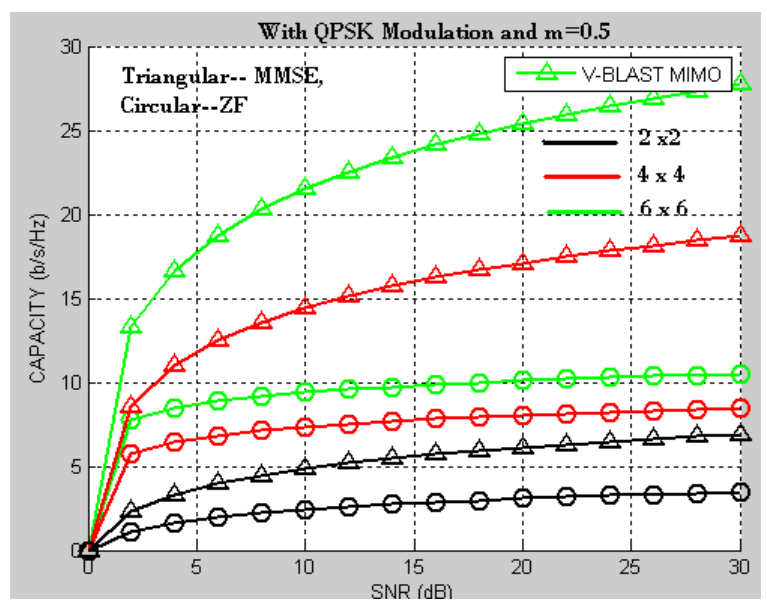

Fig. 7: Capacity vs. SNR curves for different VBLAST MIMO system in Nakagami channel $(m=0.5)$ with QPSK modulat ion.

Above figure represents a comparative analysis between the different configuration of MIMO systems in same channel condition (Nakagami channel with $\mathrm{m}=0.5$ ) and with the same digital modulation scheme. As in figure with the increase in the number of antennas in transmitter and receiver side the difference in channel capacity for MIMO systems get enhanced.

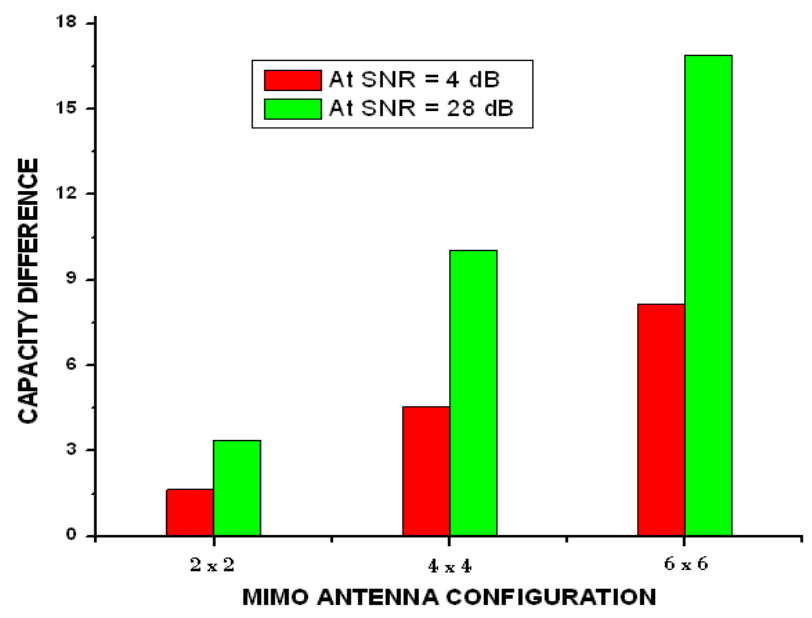

Fig. 8: Channel capacity comparison of MIMO system in lower $(1 \mathrm{~dB})$ and higher $(11 \mathrm{~dB})$ SNR region.

Figure 8 shows the variation in channel capacity with the variation in the number of antennas and signal SNR level. For a particular antenna configuration with the increment on signal SNR level the channel capacity increases accordingly 


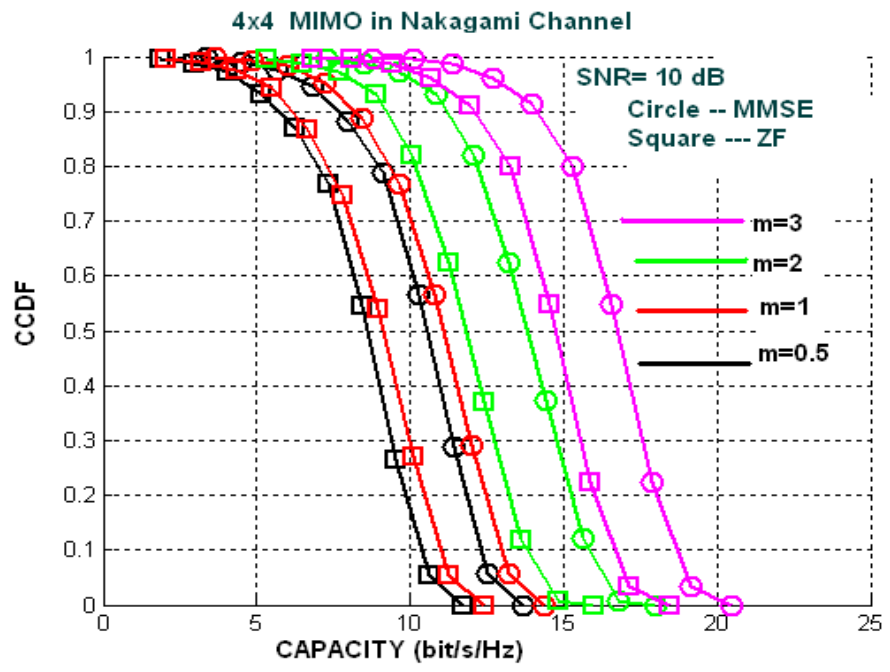

Fig 9. CCDF of capacity

Above figure 9 shows CCDF of the capacity in Nakagami channel as a function of the capacity. From the above figure one can conclude that the MMSE based receiver provides capacity gain in comparison to that of $\mathrm{ZF}$ receiver.

\section{Conclusion}

In this paper, we present the BER and capacity performance of linear receivers in MIMO Nakagami-m fading channel. We also quantify the BER and capacity performance advantage of the MMSE-VBLA ST over the ZF-VBLAST through simulation and also we find out that the simulated results follow the mathematical relation as presented in this paper. And also we find that at high SNR region MMSE and ZF receivers do not perform similarly in comparis on to the low SNR region.

\section{References}

[1] X Gu, X-H Peng and G C Zhang, "MIMO systems for broadband wireless communications", BT Technology Journal • Vol 24 No 2 • April 2006, pp-90-96

[2] G.J. Foschini. "Layered Space-Time Architecture For Wireless Communication In A Fading Environment When Using Multi-Element Antenna,” Bell Laboratories Technical Journal, pp. 41-59, Oct. 1996.

[3] J. H. Winters, "On the capacity of radio communications systems with diversity in rayleigh fading environments,” IEEE J. Select. Areas Co mmun., vol. JSA C-5, pp. 871-878, June 1987.

[4] Branka Vucetic, Jinhong Yuan, "Space-Time Coding”, John Wiley \& Sons Ltd, 2003.

[5] Yi Jiang, Xiayu Zheng and Jian Li, "Asymptotic Performance Analysis of V-BLAST", GLOBECOM, 2005.

[6] G. J. Foschini, “Layered space-time architecture for wireless communication”, Bell Labs. Technology. Journal, Vol. 6, No.3, PP. 311-335, 1998.

[7] P.W. Woln iansky, G.J Foschini, G.D. Golden, and R.A. Valenzuel "V-BLAST: An Architecture For Realizing Very High Data Rate Over The Rich-Scattering Wireless Channel,” Proc. of URS 
International Symposium on Signals, Systems and Electronics (ISSS '98), pp. 295 - 300, Sep/Oct 1998, doi: 10.1109/ISSSE.1998.738086

[8] Li Tang and Zhu Hongbo, “Analysis and Simulation of Nakagami Fading Channel with MATLA B”, CEEM-2003, China, Nov. 4-7,2003.

[9] Li Tang, Zhu Hongbo,"Analysis and Simulation of nakagami fading channel with Matlab,"AsiaPacific conference on environ mental electro magnetic CEEM'2003, China,pp. 490-494.

[10] M. Nakagami, “The m-distribution, a general formula of intensity distribution of rapid fading," in Statistical Methods in Radio Wave Propagation, W. G. Hoffman, Ed, Oxford, England: Pergamum, 1960.

[11] George K.Karagiannidis, Dimitris A.Zogas, and Stavros A. Kotsopoulos "On the Multivariate Nakagami-m Distribution With Exponential Correlation”, IEEE TRANSACTIONS ON COMMUNICATIONS, VOL.51, NO.8, AUGUST2003.

[12] A. Annamalai, and C. Tellambura, "Error Rates for Nakagami-m Fading Multi-channel Reception of Binary and M-ary Signals”, IEEE TRANSACTIONS ON COMMUNICATIONS, VOL. 49, NO.1, JANUA RY 2001.

[13] Peter Drotár, Juraj Gazda, Pavol Galajda, Dušan Kocur, “Receivers for Spatially Multiplexed MIMO Transmission Systems”, RTT, 2009.

[14] Hyundong Shin, and JaeHong Lee, "On the Error Probability of Binary and M-ary Signals in Nakagami-m Fading Channels” IEEETRANSACTIONSONCOMMUNICATIONS, VOL.52, NO.4, APRIL2004.

[15] M.K. Simon and M.-S. Alouin i, "Digital Communication Over Fading Channels :A Unified Approach to Performance Analysis”. NewYork: Wiley,2000.

[16] Zheng Du, Julian Cheng and Norman C. Beaulieu, "Asymptotic BER performance of OFDM in frequency selective Nakagami-m channels",

[17] H.V.Poor and S.Verdu, "Probability of error in MMSE multiuser detection," IEEE Transaction on Information. Theory, vol.43, pp.858-871, May1997.

[18] P.Li, D.Paul, R.Narasimhan, and J.Giiof On the distribution of SINR for the MMSE MIMO receiver and performance analysis," IEEE Transaction on Information. Theory, vol.52, pp.271-286, Jan.2006.

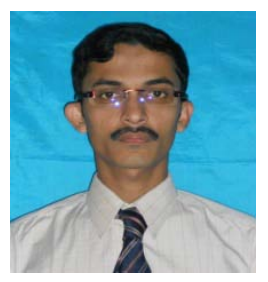

Mr. Samarendra Nath Sur: Born in 1984 at Hooghly , West Bengal, INDIA He received his M.Sc. (Electronics Science) from Jadavpur University in the year 2007 and M.Tech from Sikkim Manipal University in 2012. Currently working as an Assistant Professor in Electronics \& Communication Engineering Department of Sikkim Manipal Institute of Technology, India. Broadband Wireless Communication Advanced Digital Signal Processing and Remote Sensing are the area of specializations.

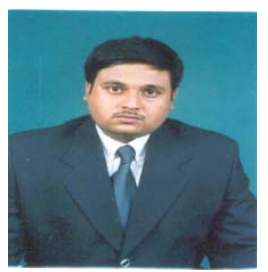

Mr. Debjyoti Ghosh: Born in 1980 at Hooghly, West Bengal, INDIA. Received B.Tech from University of Kalyani in the year 2003. Currently working as a Assistant Professor, Department of E\&C Engineering, Sikkim Manipal University, Sikkim, India. Broadband Wireless Mobile Communication, Remote Sensing and Digital Signal processing are the area of specializations. 